\title{
Biped Gait Generation Using Fourier Series at a Simulation Environment
}

\author{
F. A. Saeid Jafari Zadeh, S. B. Abbas Khosravi, T. C. Seyed Mojtaba Hosseinifar, F. D. Najme \\ Roozmand, and F. E. Abdolreza Moghimi
}

\begin{abstract}
This article contains around explanation is PersianGulf3d team activities. Focus on the main team of software such as simulation and optimization webot, MSRS algorithm and is obtained by this software, this device can be prevented rehash and the answer came sooner. In this way the initial data of the species adapted software and we Analysis and check this data by mathematical equations such as the Fourier series, equation taken to implement the program is obtained bring this article to a way to check "method to determine the coefficients" used by this team has outlines .
\end{abstract}

Index Terms-Webot, MSRS, fourier series, method to determine the coefficients.

\section{INTRODUCTION}

PersianGulf3D1 team in his official activities of Payam Noor University Shiraz early summer 2008 in soccer simulation league is began. Early activity, the main focus on the team check and improve the free code (Open Source) such as Little Green bats, Apollo, But after a few time with a weak spell and create many problems in these codes, the approach of the group presented the new code eliminates the previous problems, the code was titled, so for the first time in 2009 of the first team code written in Java that was presented, which until then was not like competitions. The later release of the new code name MagmaOffenburg based Java and object-oriented structure, the team decided to approach the use and combine this code with your team to code and new code so that a combination of two code MagmaOffenburg and PersianGulf (old) was formed and started to work on it. Among the new structure of the code, the previous solution was not so efficient opinion research and check with the company reached the conclusion that the simulation software as we use MSRS and webot. Software for simulating robot motions are webot, the new version of this software was added to the robot Nao, MSRS Similarly, the soft macro and company to hold this tournament on the Windows operating system is designed, the two angles of each joint software amounts to the Cycle to Cycle to provide us. These data after

Manuscript received January 4, 2013; revised April 26, 2013.

F. A. Saeid Jafari Zadeh and S. B. Abbas Khosravi are with the Computer Engineering Department, University of Payam Noor, Shiraz, Iran (e-mail: Kameltareen@Yahoo.com, Khosravi1356@Yahoo.com).

T. C. Seyed Mojtaba Hosseinifar is with the Industry Engineering Department, University of Payam Noor, Shiraz, Iran (e-mail: Hoseinifar.m@Gmail.com).

F. D. Najme Roozmand is with the psychology Department, University of Payam Noor, Shiraz, Iran (e-mail: Roozmandn@Yahoo.com).

F. E. Abdolreza Moghimi is with the Computer Engineering Department, University of Payam Noor, Shiraz, Iran (e-mail: Sarani797@Yahoo.com). storage were analyzed and so can benefit formula for seeking any amount of detail obtained per cycle. Mention this point is also important that these software differences and differences desired server in many competitions are different in different grade such as joints, differences in robot Height difference in relation to environment, server, robot and etc. The differences that caused the data from this software environment Simspark well do your software algorithm did not need to lose is their change and optimization, genetic algorithm one of these algorithms are.

\section{COMMENTS}

This article consists of 3 parts:

1) Activities Conducted in This Field Since

2) Activities Done by the Team Persiangulf3d

3) Activities and Future Goals Team Persiangulf3d

The following description outlines each of the above...

\section{ACTIVITIES CONDUCTED IN THIS FIELD SINCE}

Three-dimensional field simulation football simulator discussed the trends in the tournament, so far no large-old, first pioneered similar spherical robot for the first time were used, then the first generation robot having two legs and two feet high without body a text field attached to urgently while a new generation of robots to human Soccerbot full view was presented, with the coming of this little robot man competition Thumb mode towards technical football skills found early lead; after years of evolution The robot, for the first time in World Championship in 2008 CE to Nao robot was presented. The robot in terms of better flexibility to act Soccerbot but now because of restrictions that can be seen in this robot seems to many changes in the future .... The server is formed! With the New Soccerbot virtual world arena, many teams try to offer similar skills that were human, the way to the skills and the importance of higher complexity than other skills enjoyed because other skills can be provided talk directly taken from nature, but in providing these skills because of the nature of vector model of structural differences in human and robot is far away, for example following two diagrams for joints of a robot walking with a man shown in the can clearly be seen the differences. [1]

Different from the first ideas in this field were considered, by repeating the algorithm one of these ideas was the algorithm to this case, the value of each robot joint, and then was assigned values until reaching robot a walking idea $\mathrm{Al}$ was changed. At first the idea seemed very interesting, 
because in each case reached the desired answer, but what price and how long cost? Yes, this kind of view, the problem leads to the problems are NP $\mathrm{N} d$, the complexity of issues that are very long, so this requires the algorithm to a more useful and better than before were visible.

Among the use of genetic algorithm, this vacuum will give substantial coverage. Way that time complexity for solving such problems will be very low, so expanding this idea, the use of Genetic algorithm among teams participating in RoboCup simulation competition was much more pervasive. The other hand, genetic algorithm also has disadvantages such as relatively high waste Offline applications team that was due to other characteristics such as physical properties such as the use of ZMP, using methods of mathematical and simulation software to orientation [2] .

\section{ACTIVITIES DONE BY THE TEAM PERSIANGUlF3D}

As was mentioned in the introduction, the team looks after the Persian Gulf 3D football history analysis and 3D simulation, based on its work based on data adapted simulation software such as the MSRS and we bots said.

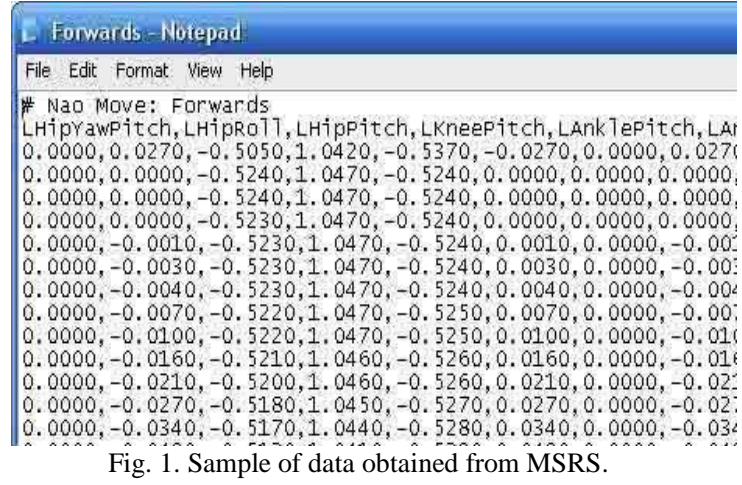

Fig. 1 Sample of data obtained from the MSRS software implementations are walking skills. The cycle to cycle View the detailed, can chart the different modes as shown in Fig. 2 obtained.

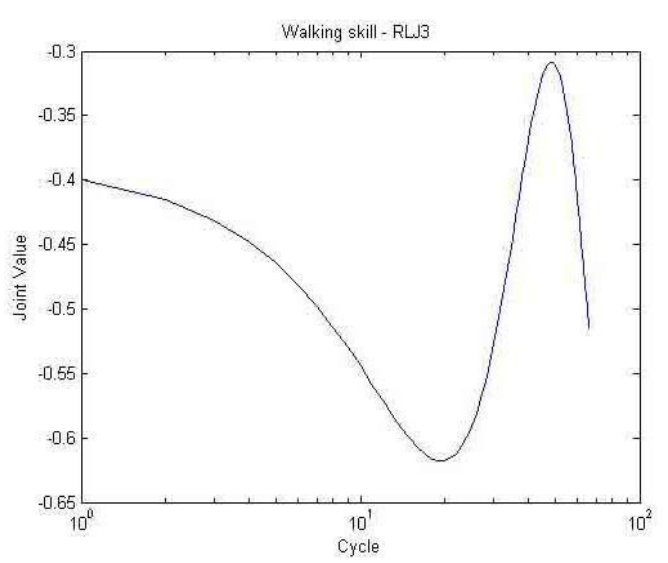

Fig. 2. Chart data obtained from a joint of left foot.

With analysis of the Fourier (Fourier series) figures by the series can be related to the trigonometric formulas for each joint separately obtained.

$$
\left(S_{N} f\right)(x)=\frac{a_{0}}{2}+\sum_{n=1}^{N}\left[a_{n} \operatorname{Cos}(n x)+b_{n} \operatorname{Sin}(n x)\right], \mathrm{N}>=1
$$

\section{A. Method to Determine the Coefficients}

Considering Fig. 2, can be clearly seen the frequency graph was obtained from this formula using the specific chart series Fourier with high approximation is as follows:

$$
f(x)=0.07877 \cos (0.1084 x)-0.1332 \sin (0.1084 x)-0.4635
$$

Top of formulas and functions Uc periodic function, we have benefited. (Uc function is multivariate.) Case above general formula is as follows. [3]

$$
f(x)=A \cos (0.1084 x)-B \sin (0.1084 x)-C
$$

As you know, in the alternative charts, change coefficients of polynomial function would change because change causes shift constant function along the axis width Game (y) and change the coefficients of variables cause changes in frequency diagram is. [4]

For example, consider the function $y=A \sin (t)+c$ as a simple periodic function, the following three figures (Fig. 3, Fig. 4 and Fig. 5) can be won.

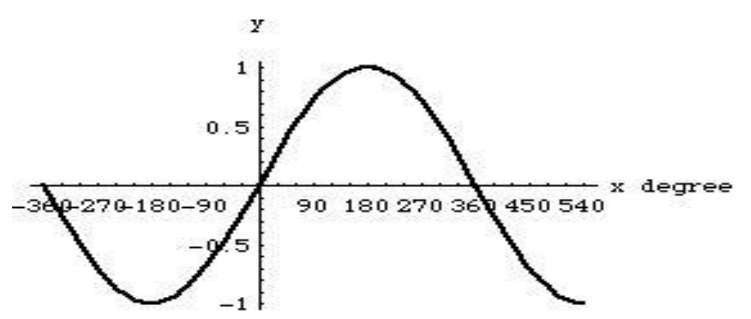

Fig. 3. Graph the function $y=\sin (0.5 x)$.

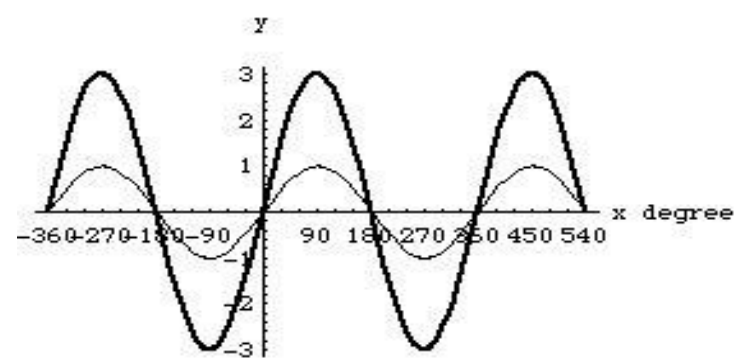

Fig. 4. Graph the function $y=3 \sin (x)$ against of previous $\operatorname{chart}(y=\sin (x))$. y

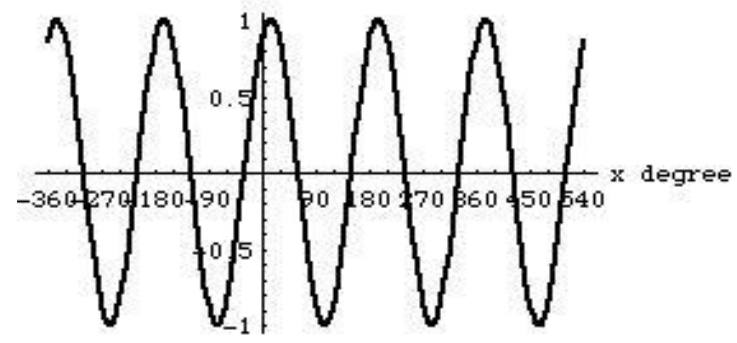

Fig. 5. Graph function $y=\sin (2 \times x+60 \times$ Degree $)$.

I.e. function can be fine for Tommy periodic functions considered and the data Webot MSRS also follow this rule is: [5], [6]

$$
f(x+\alpha)=f(x) \Leftrightarrow \text { PeriodicFunction }
$$

These property functions with alternating diagram can maintain its primary format, only the necessary parts and function changed as critical points and the better the algorithm can be implemented walking robot. For example, if 
the joint function for a particular operation as " $y=2 \sin t$ " can be combined with this method and genetic algorithm, (A, B, C) better with less time for the robot can be found.

To use this method, genetic algorithms as a chromosome in the sentences are considered. Obviously, such a coefficient of each gene is considered.

Chromosomes in a completely random initial population are selected, the method of choice here Rolette Wheel considered is because most state intersection operation with optimum 0.6 to 1 chance and mutation operation with probability 0.1 , or about occurs less so in this experiment, the intersection of 0.8 and mutation took in 0.05 [7].

Results of genetic algorithm in each test given in the relevant oefficients for the best result after traversing a given radius $R=0 / 5$ around each coefficient to be obtained. Returns values of this method, based on number of robots are useful steps; this means that robots eat without the balance to take a step forward. (Also from the point of balance is calculated GYR).

\section{Team Activities And Future Goals Persiangulf3D}

As one of the most important skills among robot skills, skills in walking robots is total balance and the severe wasting time on genetic algorithm and offline learning methods like the use of newer approaches CPG and ZMP It seems very useful. It is important to note that the use of newer methods such as "method to determine the coefficients of" genetic algorithm running time than the lower house. But the team believes, when the algorithm can be enough that it could be the online game to closed.

\section{CONCLUSION}

The advantage of the method presented in this paper is the genetic algorithm optimization. Since the focus is only on the critical points of functions 3 , so the number and also to compare their domain automatically decrease significantly. Therefore less time spent by the solution to the problem. M. on six floors this process to help the team long-term goals presented as Algorithm (online) is. I mention this point is also important that there be the optimal method to compare the number is very high (in the best population member is around 10 billion-without taking strategy genetic algorithm [NP problems]).

\section{ACKNOWLEDGMENT}

Thanks Fars Payame Noor University President Mr. doctor Moghely and Office of Research Mr. doctor Jajarmizade, His Excellency Dr. A.R.Kazemi and Mr. Rohani, who are team's supervisors.

\section{REFERENCES}

[1] L. Yang, C. Chew, T. Zielinska, and A. Poo, "A uniform biped gait generator with off-line optimization and online adjustable parameters," Robotica, Cambridge Press, vol. 25, no. 2, pp. 1-17, March 2007.

[2] "The presentation from TDP by teams participating in Robo Cup," World Championship, 2007.

[3] S. Faryab, Differential Equations Part II (Course of Mathematics), Tehran: Payam Noor University, 1382.

[4] P. Shahriar, Alternate Function, Mashhad: Mashhad publishing organization.

[5] K. Knopp, "Periodic functions," Ch. 3 in Theory of Functions Parts I and II, Two Volumes Bound as One, Part II, New York: Dover, 1996, pp. $58-92$.

[6] J. Spanier and K. B. Oldham, "Periodic functions," in An Atlas of Functions, Washington DC: Hemisphere, 1987, Ch. 36, pp. 343-349.

[7] Wikipedia. [Online]. Available: http://www.wikipedia.com

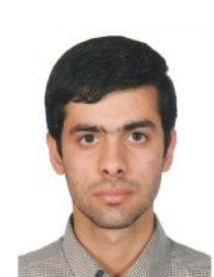

Saeid Jafari Zadeh was born in Iran in 1989. He has received the bachelor's degree in software engineering, Payam Noor University of Shiraz in 2010 and passed Master level computer engineering (artificial intelligence) in Shiraz University. His total studies had been in Iran. He is working at the university. He has presented numerous papers in the field of robotics. Jafari Zadeh has submitted numerous papers for various conferences where you can search for it.

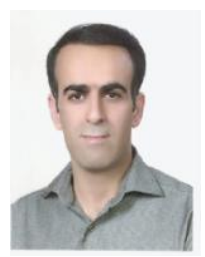

Abbas Khosravi was born in Iran in 1978. He has spent the bachelor's degree in software engineering, Shiraz University in 2000 and passed Master level IT engineering (software design and production trends) in Shiraz University. His total studies had been in Iran. He is working at the university. He has presented numerous papers in the field of robotics. He likes to study Ph.D. outside of Iran. Khosravi has submitted numerous papers for various conferences where you can search for it.

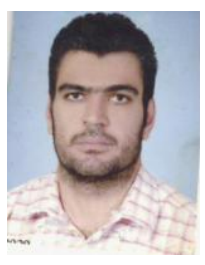

Seyed Mojtaba Hosseinifar was born in Iran in 1984. $\mathrm{He}$ has spent the bachelor's degree in industrial engineering in Azad University of Shiraz in 2008 and passed in master level management in Payam Noor University of Tehran. All of his studies had been in Iran $\mathrm{He}$ is working at the university. He likes to study Ph.D. outside of Iran. Hosseinifar has sent too many papers for various conferences that you can search for it.

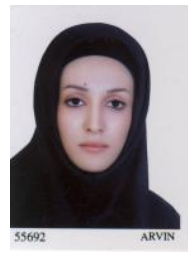

Najme Roozmand was born in Iran in 1988. She has received the bachelor's degree in psychology degree in Payam Noor University of Shiraz in 2012. All of her studies had been in Iran. She is not working. She is interested in studing Ph.D. Roozmand has sent too many papers for various conferences that you can search for it.

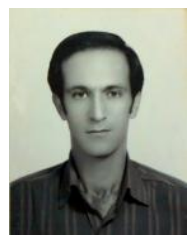

Abdolreza Moghimi was born in Iran in 1984. He has spent the bachelor's degree in software engineering, Azad University of Shiraz in 2008. All of his studies had been in Iran. He is working at the university. He has presented numerous papers in the field of robotics. Moghimi has submitted numerous papers for various conferences where you can search for it. 